\title{
Dix conseils pour améliorer ses publications sur Instagram
}

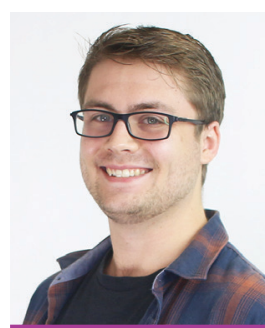

Will Cowan

En tant que créateur de contenu pour Marketing4ECPs, Will Cowan imagine et produit des vidéos pour des cabinets d'optométrie partout en Amérique du Nord. Communiquez avec Will sur le site marketing4ecps.com..

I nstagram est l'une des plateformes de médias sociaux les plus populaires, comptant près de 500 millions d'utilisateurs qui s'y connectent chaque jour. Toutefois, cette multitude de voix entraîne un défi auquel chaque cabinet d'optométrie doit faire face : se démarquer et exercer une influence. Ce ne sont pas toutes les images qui véhiculent une histoire pertinente, alors jetons un coup d'œil aux 10 conseils essentiels pour des publications sur Instagram qui se distinguent.

\section{Avoir un plan}

La première chose à faire lorsque vous commencez à publier sur Instagram est d'avoir un plan de contenu efficace. Ce plan comprendra les photos, les images et les vidéos que vous voulez présenter sur votre fil, l'esthétique qui représente votre marque ainsi que votre fréquence de publication. Ce travail préparatoire contribuera à la cohérence et à la pertinence de votre histoire ainsi qu'à sa conformité à la marque.

\section{Publier au bon moment}

Croyez-le ou non, il y a des moments meilleurs que d'autres pour publier! En général, il est préférable de publier entre $11 \mathrm{~h}$ et $13 \mathrm{~h}$ et entre $19 \mathrm{~h}$ et $21 \mathrm{~h}$, lorsque les gens sont en pause ou ont terminé le travail.

\section{Accorder la priorité au contenu vidéo}

Publier une photo, c'est super, mais publier une vidéo, c'est encore mieux. Les vidéos et les Stories vous donnent l'occasion de raconter votre histoire de façon dynamique. De plus, grâce aux options vidéo offertes par Instagram et par les téléphones modernes, il n'a jamais été aussi facile de réaliser une vidéo. Ce qui nous amène au point suivant...

\section{Toujours modifier vos photos et vos vidéos}

La concurrence est très féroce quant au contenu sur Instagram, alors si vous publiez des photos et des vidéos qui n'ont pas l'air professionnelles, votre public risque de passer directement à la prochaine publication. Prenez le temps de peaufiner votre contenu et assurez-vous qu'il est accrocheur. Parfois, le simple ajout d'un filtre peut donner d'énormes résultats.

\section{Inclure un appel à l'action}

Les légendes de photos doivent être percutantes et accrocheuses, mais ne sous-estimez jamais le pouvoir d'un appel à l'action. Pour ce faire, vous pouvez poser des questions à vos abonnés ou créer des concours et des sondages. Plus vous prolongez l'interaction d'une personne avec votre contenu, plus elle est susceptible de descendre plus bas dans l'entonnoir des ventes.

\section{Ajouter un lieu}

Vous pouvez identifier un lieu pour chacune de vos publications, ce qui vous permet de cibler les gens de votre région. Vous pouvez choisir d'être très précis, en indiquant le lieu de votre cabinet; ou très général, en identifiant votre ville.

\section{Ne pas oublier l'identification}

Si votre publication comprend un membre du personnel ou un produit, n'oubliez pas de l'identifier dans la photo et la légende. Ainsi, vous pourrez atteindre votre portée organique sans fournir de grands efforts.

8. Ne pas surcharger vos publications de motsclics Dans la plupart des cas, un ou deux motsclics pertinents suffisent dans une publication; en mettre plus peut sembler forcé. Pensez plutôt à vous concentrer sur la qualité de votre légende avant d'ajouter un motclic. Par exemple, si votre cabinet est situé à Calgary, vous pourriez ajouter \#YYC \#YYCOptométrie.

\section{9. Être authentique}

N'ayez pas peur de vous présenter tel que vous êtes dans vos publications. Les gens détectent assez bien les publications sur Instagram qui cherchent purement à vendre quelque chose; en personnalisant votre contenu, vous pouvez accroitre l'engagement du public et créer un sens de familiarité avec votre marque.

\section{S'amuser}

Enfin, n'oubliez pas de vous amuser! Instagram est une plateforme réunissant du contenu extraordinaire qui encourage l'interaction. Plus vous vous amuserez, plus vos publications seront stimulantes et engageantes. Par exemple, vous pouvez publier des photos ludiques de vos employés qui portent des lunettes de soleil ou une citation intéressante à propos de la vision.

Trouvez des exemples de bonnes publications pour votre compte Instagram professionnel en suivant certains de vos pairs qui font un travail extraordinaire. En voici quelquesuns que nous aimons beaucoup : @seeandbeseeneyecare @drmacalpineaurora2020 @terrihollandoptometry 